\title{
The chaos of the solution semigroup for some partial differential equations in weighted Banach spaces
}

Xiangdong Yang*

"Correspondence:
yangsddp@126.com
Department of Mathematics,
Kunming University of Science and
Technology, Kunming, 650093,
China

\begin{abstract}
In this paper we deal with the solution semigroup of some partial differential equations in a weighted Banach space on the real axis. We aim at showing the connection between complex-analytic approach and chaotic theory. With the approach of Carleman's formula and Joel H Shapiro's construction, we could construct dense systems of functions from which the chaos of the solution semigroup follows. The novelty of our paper is the usage of the complex-analytic approach in investigation on chaos of some partial differential equations. As far as we know, our manuscript is the first paper in this direction.
\end{abstract}

MSC: 35K30; 30E20

Keywords: partial differential equation; $C_{0}$-semigroup; weighted Banach spaces; chaos; Carleman's formula

\section{Introduction}

In [1] and [2], the author considered the following partial differential equation:

$$
\frac{\partial}{\partial t} u+c(x) \frac{\partial}{\partial x} u=h(x) u, \quad t \geq 0, x \in[0, \infty)
$$

with an initial condition

$$
u(0, x)=f(x), \quad x \in[0, \infty)
$$

where $c(x)=a$ or $c(x)=a x$ with $a$ a positive constant and $h(x)$ is a continuous bounded function on $[0, \infty)$. In case of $f(x) \in C_{0}([0, \infty))$, where $C_{0}([0, \infty))$ consists of all complexvalued functions on $[0, \infty)$ satisfying $\lim _{x \rightarrow \infty} f(x)=0$ with the norm $\|f\|=\sup _{x \in[0, \infty)}|f(x)|$, both the hypercyclicity and the chaos of the solution semigroup $\{Q(t)\}_{t \geq 0}$ of (1) and (2) in the form

$$
(Q(t))(x)=\exp \left(\int_{x}^{x+t} h(s) \mathrm{d} s\right) f(x+t)
$$

are characterized.

It is natural to ask the following question:

o2014 Yang; licensee Springer. This is an Open Access article distributed under the terms of the Creative Commons Attribution License (http://creativecommons.org/licenses/by/2.0), which permits unrestricted use, distribution, and reproduction in any medium, provided the original work is properly cited. 
Does the hypercyclicity or chaos of the solution semigroup $\{Q(t)\}_{t \geq 0}$ of (1) and (2) still hold when $f(x)$ is in other Banach spaces?

In the present paper we are concerned with the above question. Our study will be focused on the weighted Banach space $C_{\alpha}$. Let $\alpha(x)$ be a nonnegative continuous function defined on $\mathbb{R}^{+}=[0, \infty)$, henceforth called a weight, satisfying

$$
\lim _{x \rightarrow+\infty} x^{-1} \alpha(x)=\infty
$$

Given a weight $\alpha(x)$, the weighted Banach space $C_{\alpha}$ consists of complex continuous functions $f$ defined on the half real axis with $f(x) \exp (-\alpha(x))$ vanishing at infinity, and is normed by

$$
\|f\|_{\alpha}=\sup \{|f(x) \exp (-\alpha(x))|: x \in[0,+\infty)\}
$$

for $f \in C_{\alpha}$.

Following [3, 4], and [5], we define the operator $T_{a}: C_{\alpha} \rightarrow C_{\alpha}$ of translation by the complex number $a$ by

$$
T_{a} f(x)=f(x+a) \quad\left(f \in C_{\alpha}, a \in \mathbb{C}\right) .
$$

In [4], the normal family in an open set in the complex plane which is integer translates of an entire function is characterized. The hypercyclicity of bounded translation operators on Hilbert spaces of entire functions which have slow growth is characterized in [3]. The translation operators are engaged in [5] to get the intriguing and beautiful chaotic characterizations of simple connectivity. For the reader's convenience, we shall recall some basic facts on the concept of chaos.

In the last decade it has been observed that chaotic behavior in the sense of Devaney [6] can occur in some infinite-dimensional space for a linear operator. Recall a continuous linear operator $T$ on a topological vector space $X$ is called hypercyclic if there exists a vector $x \in X$ whose orbit $\left\{T^{n} x \mid n=0,1, \ldots\right\}$ is dense in $X$. A periodic point for $T$ is a vector $x \in X$ such that $T^{n} x=x$ for some $n \in \mathbb{N}$. T is said to be chaotic if it is hypercyclic and its set of periodic points is dense in $X$.

Much of the work that has been done on hypercyclic operators depends on the following hypercyclicity criterion (see [7] and [5]).

Lemma 1.1 (The hypercyclicity criterion) Suppose $T$ is an operator on a Fréchet space $X$. Suppose further that there are dense subsets $X_{0}$ and $Y_{0}$ of $X$, and a mapping $S: Y_{0} \rightarrow Y_{0}$, such that:

(a) $T^{n} \rightarrow 0$ pointwise on $X_{0}$,

(b) $S^{n} \rightarrow 0$ pointwise on $Y_{0}$,

(c) TS is the identity map on $Y_{0}$.

Then $T$ is hypercyclic on $X$.

In this paper we shall show that the solution semigroup of (1) which is defined in (3) is chaotic in some $C_{\alpha}$. Our proof is based on constructing function system which is dense and periodic under the acting of the solution semigroup, which is a totally complex-analytic approach. In Section 2, we introduce some basic results from complex analysis. Our theorem on chaos of the solution semigroup of (1) will be proved in Section 3. 


\section{Preliminary lemmas}

From now on, $A$ denotes positive constants and it may be different at each occurrence.

Let us recall Carleman's formula, which connects the zeros of a holomorphic function with its behavior on the boundary of a circle.

With a sequence of numbers $\Lambda=\left\{\lambda_{n}=\left|\lambda_{n}\right| e^{i \theta_{n}}: n=1,2, \ldots\right\}, \lambda_{n} \in \mathbb{C}$, we associate the function

$$
N_{\Lambda}(R)=\sum_{\left|\lambda_{n}\right| \leq R}\left(\frac{1}{\left|\lambda_{n}\right|}-\frac{\left|\lambda_{n}\right|}{R^{2}}\right) \cos \theta_{n} .
$$

Carleman's formula is as follows (see [8] and [9] for more details).

Lemma 2.1 Let $f(w)$ be a function analytic on $S=\{w=u+i v: \Re w \geq 0,|w| \leq R\}$, then

$$
\begin{aligned}
N_{\Lambda}(R)= & \frac{1}{\pi R} \int_{0}^{\pi} \log \left|f\left(R e^{i \theta}\right)\right| \cos \theta \mathrm{d} \theta \\
& +\frac{1}{2 \pi} \int_{0}^{R}\left(\frac{1}{v^{2}}-\frac{1}{R^{2}}\right) \log |f(i v) f(-i v)| \mathrm{d} v+d_{f}(R),
\end{aligned}
$$

where $N_{\Lambda}(R)$ is the function associated with the zeros off $(w)$ in $S$ defined by (5) and $\left\{\theta_{n}\right\}$ is the corresponding sequence of arguments. Furthermore, $d_{f}(R)$ is a function of $R$ depends on $f$, satisfying

$$
d_{f}(R)=O(1) \quad \text { as } R \rightarrow \infty
$$

It will be important for us to investigate the denseness of some particular systems of functions in the $C_{\alpha}$. The basic idea of the following lemma originates from $[10,11]$ and [12].

Let $\alpha(x)$ be a positive continuous function on the half-axis, if for every fixed $R \geq 0$, the quantity

$$
\alpha^{*}(R)=\sup _{x \geq 0}\{R x-\alpha(x)\}
$$

is finite. It is called the Legendre transform or the Young dual function for $\alpha$ (see [9]).

Denote by

$$
M_{\alpha^{*}}(R)=\frac{1}{2 \pi} \int_{1}^{R}\left(\frac{1}{v^{2}}-\frac{1}{R^{2}}\right) \frac{\alpha^{*}(4|v|)}{2} \mathrm{~d} \nu .
$$

Lemma 2.2 Let $\alpha(x)$ be a nonnegative continuous function satisfying (4), let $\Lambda=\left\{\lambda_{n}=\right.$ $\left.\left|\lambda_{n}\right| e^{i \theta_{n}}: n=1,2, \ldots\right\}$ be a sequence of complex numbers satisfying $\Re \lambda_{n}>0$, furthermore, let $N_{\Lambda}(R)$ be the function associated to $\Lambda$ defined by (5), $\alpha^{*}(R)$ and $M_{\alpha^{*}}(R)$ be defined by (6) and (7) separately. If

$$
\limsup _{R \rightarrow \infty}\left(N_{\Lambda}(R)-\frac{\alpha^{*}(R)}{R}-M_{\alpha^{*}}(R)\right)=\infty,
$$

then the system $\left\{e^{\lambda_{n} x}\right\}$ is dense in $C_{\alpha}$. 
Proof We use the Hahn-Banach theorem. Suppose $\phi$ is a continuous linear functional on $C_{\alpha}$ that annihilates each exponential function $\left\{e^{\lambda_{n} x}\right\}$ for $\lambda_{n} \in \Lambda$. By Hahn-Banach we will be done if we can show that $\phi=0$ on $C_{\alpha}$.

The Riesz representation theorem provides a complex measure $\mu$ satisfying

$$
\|\mu\|=\int_{0}^{+\infty} e^{\alpha(x)}|\mathrm{d} \mu(x)|=\|\phi\|
$$

such that

$$
\phi(f)=\int_{0}^{+\infty} f(x) \mathrm{d} \mu(x)
$$

for $f \in C_{\alpha}$. In particular,

$$
\phi\left(e^{\lambda_{n} x}\right)=\int_{0}^{+\infty} e^{\lambda_{n} x} \mathrm{~d} \mu(x)
$$

for each $\lambda_{n} \in \Lambda$. Now the last equation shows that the function defined on the complex plane by

$$
\Phi(w)=\phi\left(e^{w x}\right)=\int_{0}^{+\infty} e^{w x} \mathrm{~d} \mu(x) \quad(w=u+i v) \in \mathbb{C}
$$

is holomorphic in the closed right half plane $\mathbb{C}_{+}=\{w: \Re w \geq 0\}$, satisfying

$$
\Phi\left(\lambda_{n}\right)=0
$$

for each $\lambda_{n} \in \Lambda$.

By the definition of $\mu$, we have

$$
|\Phi(w)|=\left|\int_{0}^{+\infty} e^{w x} \mathrm{~d} \mu(x)\right| \leq\|\mu\| \cdot e^{\alpha^{*}(|w|)} .
$$

Thanks to Lemma 2.1, we will see that (8) verifies Lemma 2.2. Consider $\Phi(w)$ in the closed half circle $S=\{w: \Re w \geq 0,|w| \leq R\}$. Without loss of generality, we can suppose that $\left|\lambda_{n}\right|>1$. Application of Carleman's formula in Lemma 2.1 yields

$$
N_{\Lambda}(R) \leq \frac{\alpha^{*}(R)}{R}+M_{\alpha^{*}}(R)+d_{f}(R) .
$$

Recall the function $d_{f}(R)$ defined in Lemma 2.1 remains bounded as $R \rightarrow \infty$. This forces

$$
\limsup _{R \rightarrow \infty}\left(N_{\Lambda}(R)-\frac{\alpha^{*}(R)}{R}-M_{\alpha^{*}}(R)\right)<\infty,
$$

which gives $\Phi(w) \equiv 0$, proving Lemma 2.2 .

For a function $f(z)$ regular in the right half plane $\Re z \geq 0$, the indicator function of $f(z)$ is defined by (see [8])

$$
h(\theta)=\limsup _{r \rightarrow \infty} r^{-1} \log \left|f\left(r e^{i \theta}\right)\right|, \quad|\theta| \leq \frac{\pi}{2} .
$$


We also need the following uniqueness theorem on holomorphic functions of exponential type growth on the right half plane characterized by the indicator functions (see [8]).

Lemma 2.3 (Carleson's theorem) Suppose that $f(z)$ is regular and exponential type in $\Re z \geq 0$ and $h\left(\frac{\pi}{2}\right)+h\left(-\frac{\pi}{2}\right)<2 \pi ;$ then $f(z) \equiv 0$ if and only if $f(k)=0, k=1,2, \ldots$.

\section{The chaotic theorem}

With the useful criteria for the density in Lemma 2.2 and Lemma 2.3 in hand, we are able to prove Theorem 3.1, which is the main result of this paper.

Theorem 3.1 Let $Q(t)$ be the solution semigroup of (1) defined in (3) with $f(x) \in C_{\alpha}$, where $x \in[0,+\infty)$ and $h(x)$ is a bounded continuous function. If

$$
\exp \left(\int_{x}^{x+t} h(s) \mathrm{d} s\right)=\exp \left(\int_{x}^{x+t+a} h(s) \mathrm{d} s\right)
$$

holds for some positive constant a, then the discrete semigroup $\{Q(n a)\}_{n=1}^{\infty}$ is chaotic in $C_{\alpha}$.

Proof It is obvious that the discrete semigroup $\{Q(n a)\}_{n=1}^{\infty}$ is very close to the translation operators $T_{a}$. We shall follow the proof for $T_{a}$ in [5]. We will proceed with the proof in two steps.

Step 1: The solution semigroup $Q_{a}$ is hypercyclic in $C_{\alpha}$.

Our business is to find the dense subspaces $X_{0}$ and $Y_{0}$ and the inverting operator $S$ required by the hypercyclicity criterion in Lemma 1.1.

Let us define

$$
F_{-}=\left\{e^{\lambda_{j} x}: \Re \lambda_{j}<0 \text { and } \lambda_{j} \in \Lambda \text {, where } \Lambda \text { satisfies }(8), j=1,2, \ldots\right\}
$$

and

$$
F_{+}=\left\{e^{\lambda_{j} x}: \Re \lambda_{j}>0 \text { and } \lambda_{j} \in \Lambda \text {, where } \Lambda \text { satisfies }(8), j=1,2, \ldots\right\} .
$$

By the density Lemma 2.2, it is obvious that $F_{+}$is dense in $C_{\alpha}$. The case of $F_{-}$can be done in a similar fashion, that is, applying Carleman's formula in Lemma 2.1 to the closed half circle on the left half plane $S_{-}=\{w: \Re w \leq 0,|w| \leq R\}$. This argument works as well for Lemma 2.1 and Lemma 2.2. Thus, we have obtained the dense subspaces of $C_{\alpha}$.

Let us verify the hypercyclicity criterion for the dense subset $F_{+}, F_{-} \subset C_{\alpha}$ :

(a) For every $e^{\lambda_{j} x} \in F_{-}, Q_{a}^{n} e^{\lambda_{j} x}=\exp \left(\int_{x}^{x+a} h(s) \mathrm{d} s\right) e^{\lambda_{j} x+n a \lambda_{j}}$. Note that $e^{n a \lambda_{j} x}$ can be written as $e^{n a \lambda_{j} x}=e^{\lambda_{j} x}\left(\cos \left(n a \lambda_{j}\right)+i \sin \left(n a \lambda_{j}\right)\right)$, therefore, $\lim _{n \rightarrow \infty} Q_{a}^{n} e^{\lambda_{j} x}=0$.

(b) Let $S_{a}$ be the operator translation by $-a$, i.e. $S_{a}=Q_{a}^{-1}$. For every $e^{\lambda_{j} x} \in F_{+}$, $S_{a}^{n} e^{\lambda_{j} x}=\exp \left(\int_{x-a}^{x} h(s) \mathrm{d} s\right) e^{\lambda_{j} t-n a \lambda_{j}}$. Note that $e^{-n a \lambda_{j} x}$ can be written as $e^{-n a \lambda_{j}}=e^{-n a \Re \lambda_{j}}\left(\cos \left(-n a \mathfrak{\Im} \lambda_{j} x\right)+i \sin \left(-n a \mathfrak{s} \lambda_{j} x\right)\right)$, therefore, $\lim _{n \rightarrow \infty} S_{a}^{n} e^{\lambda_{j} x}=0$.

(c) Finally, we have $Q_{a} S_{a}=I$ on $F_{+}$where $I$ is the identity operator.

Step 2: $C_{\alpha}$ admits a dense periodic points subset.

Since the obvious periodic points $e^{\lambda x}$ for $a \lambda=2 \pi i q$, where $q$ is a (real) rational number, no longer span a dense subspace of $C_{\alpha}$, it requires a bit more work. 
Denote by $E=\left\{\frac{1}{(x+a n)^{k}}, k=1,2, \ldots\right\}$. Since $\alpha(x)$ satisfies (4), we know that $E$ is a subset of $C_{\alpha}$. We are going to show that $E$ is also dense in $C_{\alpha}$. We proceed with the proof in Lemma 2.2 word by word. Denote by $\Phi(w)$ the function induced by the bounded linear functional $\phi$, then

$$
\Phi(w)=\phi\left(\frac{1}{(x+n a)^{w}}\right)=\int_{0}^{+\infty} \exp \left(\int_{x}^{x+a} h(s) \mathrm{d} s\right) \frac{1}{(x+n a)^{w}} \mathrm{~d} \mu(x) .
$$

Denote by $w=u+i v=r e^{i \theta}$, then for fixed $n$, we have

$$
|\Phi(w)| \leq A \sup _{\theta \in[0,2 \pi)} e^{-r \cos \theta}
$$

where $A$ is some positive constant depend on $n$. Thus $\Phi(w)$ is regular and exponential type in the closed right half plane $\mathbb{C}_{+}=\{w: \Re w \geq 0\}$, satisfying $h\left(\frac{\pi}{2}\right)+h\left(-\frac{\pi}{2}\right)<2 \pi$. By Lemma 2.3, we can deduce $\Phi(w) \equiv 0$ from $\Phi(k)=0$ for all $k \in \mathbb{N}$. Thus $E$ is dense in $C_{\alpha}$.

Now we proceed to construct dense periodic subset of $C_{\alpha}$ with the help of $E$. We claim that for each point $\lambda$ of the unit circle, the series

$$
\sum_{n=0}^{+\infty} \frac{\lambda^{n}}{(x+n a)^{k}}
$$

converges in the norm of $C_{\alpha}$ to an eigenvector $f$ of $Q_{\lambda}$ corresponding to the eigenvalue $\lambda$. Since $k \geq 2$, for fixed $x \in \mathbb{R}^{+}$, we have

$$
(x+n a)^{-k}=O\left(|n|^{-k}\right) \quad \text { as } n \rightarrow+\infty .
$$

Thus the absolute series

$$
\sum_{n=0}^{+\infty} \int_{0}^{+\infty} \exp \left(\int_{x}^{x+a} h(s) \mathrm{d} s\right) \frac{\lambda^{n}}{(x+n a)^{k}} \mathrm{~d} \mu(x)
$$

converges.

Let $E_{\Sigma}$ denote the collection of all these eigenvectors where $\lambda$ is a root of unity, it is obvious that $E_{\Sigma}$ is a set of periodic points of $Q_{a}$. As aforementioned, the set

$$
E_{0}=\left\{e^{\lambda x}: a \lambda \text { is a root of unity }\right\}
$$

is another collection of periodic points for $Q_{a}$. Thus the linear span of $E_{0} \cup E_{\Sigma}$ also consists entirely of periodic points. To prove Theorem 3.1, it remains to show that $E_{0} \cup E_{\Sigma}$ is dense in $C_{\alpha}$. We will see that it reduces to show that $E_{\Sigma}$ is dense in $H_{\alpha}$.

Recall that we have proved the density of the systems $E$. So it is just a job for the Hahn-Banach theorem to show that the closure of the span of $E_{\Sigma}$ contains $E$. Suppose $\phi$ is a nontrivial bounded linear functional that annihilates every function in $E_{\Sigma}$. By the Hahn-Banach theorem it is enough to prove that $\phi$ also annihilates every function in $E_{\Sigma}$. That is, we are assuming that $\phi(f)=0$ for all $|\lambda|=1$ and we want to prove $\phi\left(\exp \left(\int_{x}^{x+a} h(s) \mathrm{d} s\right) \frac{1}{(x+n a)^{k}}\right)=0$ for $n=0,1,2, \ldots$ 
Denote by $a_{n}(x)=\exp \left(\int_{x}^{x+a} h(s) \mathrm{d} s\right) \frac{1}{(x+n a)^{k}}$ and write

$$
b_{n}=\phi\left(a_{n}\right)
$$

for which we will prove are zero. Since $\phi$ is continuous and the series in (10) converges to $f$ in the norm of $C_{\alpha}$, it follows that

$$
\sum_{n=0}^{+\infty} b_{n} \lambda^{n}=\phi(f)=0 \quad(\text { all }|\lambda|=1)
$$

We will show that the left-hand side of (11) has square-summable coefficients. Thus we can deduce all of the coefficients $b_{n}$ must be zero by the uniqueness theorem from the $L^{2}$ theory of Fourier series.

By the definition of the continuity of a linear functional, there exists some positive constant $A$ such that

$$
|\phi(f)| \leq A\|f\| \quad\left(f \in C_{\alpha}\right) .
$$

In particularly, (4) yields

$$
\left|b_{n}\right|=\left|\phi\left(a_{n}\right)\right| \leq A\left\|a_{n}\right\| \leq \frac{A_{1}}{|n|^{2 k}}
$$

for all $n=0,1,2, \ldots$, where $A_{1}=A \int_{0}^{+\infty} x^{2} \mathrm{~d} \mu(x)$. Since $k>1$, we have $\sum_{n=0}^{+\infty}\left|a_{n}\right|^{2}<\infty$, which show that the right-hand side of (11) belongs to $L^{2}$ of the unit circle. Thus by (11) all the coefficients $a_{n}$ must be zero, proving Theorem 3.1.

\section{Competing interests}

The author declares that they have no competing interests.

\section{Acknowledgements}

The author gratefully acknowledges the help of the referees and the editors, which lead to the improvement of the original manuscript. His thanks also goes to Prof. Hui Fang who introduced the work of Fukiko Takeo to the author. This work was supported by National Natural Science Foundation of China (Grant No. 11261024).

Received: 18 November 2013 Accepted: 1 April 2014 Published: 06 May 2014

\section{References}

1. Takeo, F: Chaos and hypercyclicity for solution semigroups to some partial differential equations. Nonlinear Anal. 63, 1943-1953 (2005)

2. Takeo, F: Chaotic or hypercyclic semigroups on a function space $C_{0}$ or $L^{P}(I, C)$. SUT J. Math. 41(1), 43-61 (2005)

3. Chan, KC, Shapiro, JH: The cyclic behavior of translation operators on Hilbert spaces of entire functions. Indiana Univ. Math. J. 40, 1421-1449 (1991)

4. Kim, JH, Rubel, LA: Integer translation of meromorphic functions. Trans. Am. Math. Soc. 349, 1447-1462 (1997)

5. Shapiro, JH: Simple connectivity and linear chaos. Rend. Circ. Mat. Palermo Suppl. 56, 27-48 (1998)

6. Devaney, RL: An Introduction to Chaotic Dynamical Systems. Addison-Wesley, New York (1989)

7. Shapiro, JH: Composition Operators and Classical Function Theory. Springer, New York (1991)

8. Boas, RP Jr.: Entire Functions. Academic Press, New York (1954)

9. Levin, BY: Lectures on Entire Functions. Cambridge University Press, New York (1987)

10. Deng, GT: Incompleteness and closure of a linear span of exponential system in a weighted Banach space. J. Approx. Theory 125, 1-9 (2003)

11. Yang, XD: Incompleteness of exponential system in the weighted Banach space. J. Approx. Theory 153, 73-79 (2008)

12. Yang, XD, Tu, J: On the completeness of the system $\left\{t^{\lambda_{n}}\right\}$ in $C_{0}(E)$. J. Math. Anal. Appl. 368, 429-437 (2010) 